\title{
The polarizability of organometallic bonds
}

\author{
Piero Macchi ${ }^{\mathrm{a} *}$, Anna Krawczuk ${ }^{\mathrm{b}}$ \\ ${ }^{a}$ Department of Chemistry and Biochemistry, University of Bern, Freiestrasse 3, 3012 Bern, Switzerland. \\ b Faculty of Chemistry, Jagiellonian University, Ingardena 3, 30-o6o Krakow, Poland. \\ Corresponding Author: Piero Macchi, Tel: ++41 (0)31 63142 81, Fax: ++41 (0)31 63139 96, E-mail - \\ piero.macchi@dcb.unibe.ch
}

\begin{abstract}
The distributed atomic polarizabilities enable the investigation of the coordination of organic ligands to transition metals giving new insight of some organometallic complexes used in catalytic processes. This approach is useful to appreciate the enormous changes occurring upon complexation to a metal, which is mainly responsible for the augmented reactivity of these species. The method we have developed allows calculating the polarizability of an atom in a molecule upon a partitioning of the electron density, for example using the Quantum Theory of Atoms in Molecules. The polarizabilities result from numerical differentiation of the atomic dipole moments with respect to the electric field. From the polarizability tensors, other useful quantities derive, for example a mathematically precise definition of bond polarizability. In terms of chemical reactivity, the distributed polarizabilities are complementary to the traditional analysis of the electrostatic potential, which informs only on susceptibility towards hard, charge-controlled chemical reactions. On the contrary, atomic polarizabilities enable addressing the sites more keen on soft, orbital-controlled reactions.
\end{abstract}

Keywords: Electron Density; Polarizabilities; Organometallic Chemistry; Atoms in Molecules

\section{Graphical abstract}
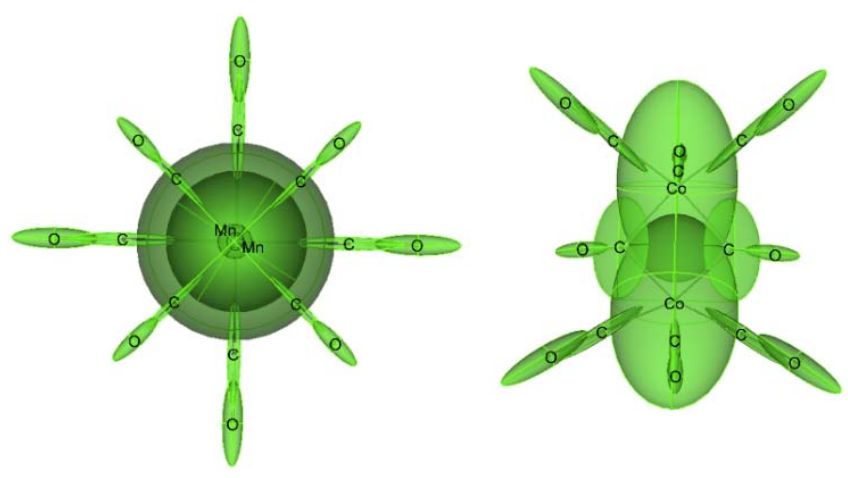


\section{Introduction.}

First and higher order electric polarizabilities $(\boldsymbol{\alpha}, \boldsymbol{\beta}, \ldots)$ of a molecule or a crystal provide essential information to understand and predict how matter interact with external electric fields. For example, the first order polarizability directly connects the electron density distribution with linear optical properties, like the refraction of light, or with the intensities of Raman scattering.

In addition, the polarizabilities reflect the attitude towards an incoming reactant. In fact, the interactions between molecules initially produce a repolarization of the electron density that could first modify the active electrostatic forces, then the type of aggregation, and eventually the reaction intermediates.

Polarizability was invoked by Pearson [1] to explain, at least in part, the reasons of softness in the well-known theory of hard and soft acids and bases. While polarizability alone is not a direct measure of the chemical hardness $\eta$ [2], it is certainly a direct manifestation of it, because molecules with small polarizability and high charge are more likely hard, whereas molecules with small charge and large polarizability usually fall in the soft class. In the strict definition,

$$
\eta=\frac{1}{2}\left(\frac{\partial^{2} E}{d N^{2}}\right)_{v}
$$

where $\mathrm{E}$ is the energy of a system of $\mathrm{N}$ electrons, $v$ the external potential, $\eta$ is not really measurable, unless approximated with finite differences,

$$
\eta=\frac{1}{2}(I-A)
$$

where I is the ionization potential and A the electron affinity) [3]. Noteworthy, the factor $1 / 2$ in equation (1) is completely arbitrary and it could be dropped, as later proposed by Pearson [4]. In this view, one can associate the concept of softness with the ability of a molecule to mix the ground with the excited states. Often this is inferred just from the evaluation of the HOMO-LUMO gap of a single determinant molecular orbital calculation. Because the molecular polarizability is itself due to a mixing of the electronic ground state with excited states, a molecule with large polarizability is softer. Recent developments in this field are those presented by Komorowski et al. [5], who correlated the Fukui functions with the polarizability, offering a theoretical tool to investigate chemical reactivity. In keeping with the original discussion by Pearson [1], a chemist would prefer to breakdown the hardness or softness $(S=1 / \eta)$ in terms of atomic or functional group contribution. Thus, the possibility to calculate distributed atomic polarizabilities is extremely useful. Moreover, being tensors, polarizabilities contain information about the anisotropy of a given interaction and therefore they could inform not only on the most favourable sites for an electrophilic/nucleophilic attack but also on the more favourable paths.

In view of their important role in chemistry, we have undertaken a project intended to calculate the atomic polarizabilities in order to investigate the role of functional groups in building up a molecular property. For example, atomic polarizabilities, calculated from a molecular electron density, allow to rapidly estimating the susceptibilities of a crystal packing of that molecule. By testing several packing 
motifs, one could screen the role of various functional groups in producing materials with specific optical properties, like high refraction, low birefringence, high second harmonic generation (if using second order polarizabilities), etc.

At the same time, this method enables to predict the attitude of a given functional group in binding or reacting with others. In this context, the investigation of the polarizability of chemical bonding (hereinafter bond polarizability) is extremely important.

In order to obtain atomic polarizabilities it is necessary to apply a partitioning of the molecular or crystal tensors, which requires a definition of atomic volumes. One method is the topological partitioning of the quantum theory of atoms in molecules QTAIM [6]. By using the scheme proposed by Keith [7], atomic polarizabilities are calculated numerically from derivation of the QTAIM atomic dipole moments with respect to the electric field. This approach, described in Krawczuk et al. [8], allows to easily obtain symmetrized atomic polarizability tensors.

For the purposes mentioned above, the atomic polarizabilities must be exportable (meaning that atoms of the same type in similar chemical environment must have a very similar $\boldsymbol{\alpha}$ ) and should be easy to be represented as tensors. Both these requirements are fulfilled, as demonstrated by our previous work [8].

In particular, the atomic tensors are fully exportable because the origin dependence is removed by applying Keith's suggestion [7]. This enables a sensible comparison of the same functional groups when embedded in different molecular environments.

In this paper, we propose to use the atomic polarizabilities and the bond polarizabilities in connection with molecular reactivity. In particular, our aim is to provide a frame that is complementary with respect to the universally adopted electrostatic potential $\phi(\mathbf{r})$. In fact, $\phi(\mathbf{r})$ addresses the sites more keen to interactions with hard nucleophiles or electrophiles (charge-controlled reactions). On the other hand, the atomic polarizabilities provide a picture of the soft sites (orbital-controlled reactions), because they anticipate the ability of an atom to repolarize upon interaction with the external field created by an incoming reactant. Noteworthy, the two approaches are not in contradiction, but simply complementary, as chemical reactions are never entirely charge- or orbital-controlled.

In the following, we first give an overview of the methods and details of the calculations and then we report calculations on some prototype organometallic molecules, characterized by most common coordination modes, like olefin complexes, carbonyl complexes, metal-metal bonds, metal-hydride, and agostic interaction.

\section{Background: the distributed atomic polarizabilities and bond polarizability within QTAIM.}

Calculating polarizabilities of atoms in molecules is a long-standing issue. Several models have been proposed in the literature [9], mostly based on classical electrostatic of point charges or multipoles, although a few of them are based on QTAIM partitioning [9c, 9d]. The main stimulus for such a 
research has always been the calculation of optical properties, intermolecular energies or solvation effects. Following the theory of atomic properties suggested by Keith [7], we have recently proposed [8] a way to calculate distributed atomic polarizabilities from molecular electron densities, using the QTAIM partitioning. In a system of $\mathrm{N}_{\mathrm{a}}$ atoms, the atomic dipole moment is:

$$
\mu(\Omega)=-\int_{\Omega}\left[r-R_{\Omega}\right] \rho(r) d r+\sum_{\Omega^{\prime}}\left[R_{\Omega}-R_{b}\left(\Omega \mid \Omega^{\prime}\right)\right] Q\left(\Omega \mid \Omega^{\prime}\right)
$$

where $Q\left(\Omega \mid \Omega^{\prime}\right)$ is the charge contributed to atom $\Omega$ by the bond to atom $\Omega^{\prime}, R_{\Omega}$ is the position vector of atom $\Omega$ with respect to an arbitrary origin and $R_{b}\left(\Omega \mid \Omega^{\prime \prime}\right)$ is the positional vector of the bond critical point between atom $\Omega$ and $\Omega^{\prime} . \mu(\Omega)$ can be considered as the summation of $\mu_{p}(\Omega)$, the atomic polarization and $\mu_{c}(\Omega)$, a charge translation. $\mu_{p}(\Omega)$ is the integration of the dipolar density function $(r \rho(r))$ within the atomic basin, whereas $\mu_{c}(\Omega)$ is given by the weighted sum of atomic charge translations from the atom's nucleus to each bond critical point (BCP) connected to that atom. The charge translation is computed from a system of equations, where some constraints must be defined. The total charge is the sum of all bond charges:

$$
Q(\Omega)=\sum_{\Omega^{\prime} \prime \neq \Omega}^{N_{a}} Q\left(\Omega \mid \Omega^{\prime}\right)
$$

where $Q\left(\Omega \mid \Omega^{\prime}\right)=0$ if $\Omega$ and $\Omega^{\prime}$ are not bonded. Furthermore, for each bond:

$$
\mathrm{Q}\left(\Omega \mid \Omega^{\prime}\right)+\mathrm{Q}\left(\Omega^{\prime} \mid \Omega\right)=0
$$

If a ring $R$ is present, the bond charges within the ring sum up to zero, though each bond is weighted:

$$
\sum_{\Omega \in R} \lambda(\Omega \mid \Omega+1) \cdot Q(\Omega \mid \Omega+1)=0
$$

where $\Omega$ is an atom belonging to the ring $R$, and $\Omega+1$ is the next atom in the ring. A sensible definition of the coefficient $\lambda(\Omega \mid \Omega+1)$ is the inverse of the electron density at the bond critical point (BCP):

$$
\lambda(\Omega \mid \Omega+1)=\frac{1}{\rho(\Omega \mid \Omega+1)}
$$

In fact, a large $\lambda$ implies a small influence of the associated bond charge therefore weaker bonds are included in equation (6) with larger $\lambda$. Equations (4), (5) and (6) produce $\left(N_{a}+N_{b}+N_{R}-1\right)$ linearly independent equations, where $N_{b}$ is the number of bonds and $N_{R}$ the number of rings. Bond charges result from the solution of this set of equations and they define the charge translation terms of atomic dipole moments and thus contribute to the total atomic dipole moments. Noteworthy, with this definition, no term of the molecular dipole moment is origin dependent. This is true also for charged molecules, although one should not think that this removes the intrinsic ambiguity of the dipole moment for ions. In fact, in non-neutral systems, the total dipole moment, computed from the bond and atomic dipole moments of equation (3), is origin independent but not invariant with respect to the partitioning scheme (hence the set of atomic charges). A partition different from QTAIM would 
produce a different total dipole moment, though origin independent. On the contrary, in neutral molecules the total dipole is both origin and partition independent.

Atomic polarizabilities are eventually obtained as numerical derivatives of the corresponding atomic dipole moments with respect to applied field:

$$
\alpha_{i j}(\Omega)=\frac{\mu_{i}^{\varepsilon_{j}}(\Omega)-\mu_{i}^{0}(\Omega)}{\varepsilon_{j}}
$$

where $\mu_{i}^{F_{j}}(\Omega)$ is the atomic dipolar component along the $i$ direction computed with a given electric field $(0$ or $\varepsilon)$ in direction $j$. The slightly asymmetric tensors are symmetrized taking the average between the tensor and its transposed matrix, as proposed by Nye [10].

Having derived atomic polarizabilities, it seems obvious to try also a definition of bond polarizability, a concept often used in chemistry but lacking a precise mathematical definition.

The term bond polarizability has been extensively adopted in the field of Raman scattering, of course because intensities depend on the derivative of polarizability with respect to a normal coordinate. Placzek [11] introduced a semiclassical approach to calculate Raman intensity based on bond polarizability that was assumed to be function only of the positions of nuclei. The molecular polarizability was then expressed as a sum of bond polarizabilities. Following this idea many research groups [12] assigned to each bond a polarizability tensor with one of the principal axes directed along the bond (longitudinal polarizability), whereas other two principal components are perpendicular (transverse polarizability). Furthermore, each bond is assumed to have cylindrical symmetry, following the assumption that the values of the principal polarizabilities of a particular bond are independent of the chemical environment. In other words, change of a bond orientation is not affecting bond polarizability components, but it affects overall molecular polarizability because these are explicit functions of bond orientations.

In many circumstances, chemists speak about bond polarizability, but they actually mean bond softness, which is not the same and it would require an appropriate definition. Nevertheless, it is obvious from what reported in the introduction that bond polarizability can be in fact associated with softness of the chemical bond.

Having quite precise atomic polarizability tensors, it is easy to define a bond polarizability as the projection of the two atomic polarizability along the direction of $\Omega-\Omega^{\prime}$ bond (here defined by the unit vector $\left.\mathbf{r}_{\Omega \Omega^{\prime}}\right)$ :

$$
\alpha_{\Omega-\Omega^{\prime}}=\mathbf{r}_{\Omega \Omega^{\prime}}^{\mathrm{T}} \cdot\left(\boldsymbol{\alpha}_{\Omega}+\boldsymbol{\alpha}_{\Omega^{\prime}}\right) \cdot \mathbf{r}_{\Omega \Omega^{\prime}}
$$

At variance from other definitions, $\alpha_{\Omega-\Omega^{\prime}}$ comes exactly from the calculated distributed atomic polarizabilities of the system and it would be a quantum mechanical observable (albeit not measurable in practice). 


\section{Computational details.}

All molecular structures were calculated with Gaussian09 [13] at B3LYP [14] level of theory using 6$311+\mathrm{G}(2 \mathrm{~d}, 2 \mathrm{p})$ basis set [15], but for compounds containing atoms with $\mathrm{Z}>36$, for which the allelectron DGDZVP [16] basis set was adopted. For an unbiased comparison with $\left[\mathrm{Ag}(\mathrm{CO})_{2}\right]^{+}, \mathrm{Cr}(\mathrm{CO})_{6}$ and CO were also calculated with DGDZVP basis set. Molecular geometries were first optimized and then wavefunctions were calculated under electric fields in $\mathrm{x}, \mathrm{y}$ and $\mathrm{z}$ directions (positive and negative). QTAIM analysis was performed on all the electron densities using AIMAll [17], in order to calculate atomic charges and dipole moments. The program PolaBer [8a] was used to calculate the atomic polarizabilities. PolaBer was also used for the visualization of the atomic tensors, which are represented in unit of volume, after applying a proper scaling for visualization purposes.

\section{Results and discussion}

\subsection{Metal carbonyls}

Metal carbonyl complexes, $\mathrm{M}(\mathrm{CO})_{\mathrm{n}}$ are among the most studied metal complexes and they are often taken as models for accurate studies or as benchmarks to test new theories on metal organic bonding. They have been investigated also with experimental or theoretical electron density analyses [18]. The bonding is classically described by the Dewar-Chatt-Duncanson model of donation and back-donation [19]. This mechanism modifies the electron density distribution of the ligand, although the stereochemistry of coordination is more important for the polarizability. Carbonyls are always end-on attached (apart in polymetal clusters) with the $\mathrm{C}$ atom directly bounded to the metal. Both $\mathrm{C}$ and $\mathrm{O}$ polarizabilities are therefore prolated with the main axis of their tensors oriented towards the metal. Of course, the $\mathrm{C}$ atom is more affected, being closer; nevertheless, the oxygen is also quite modified. Because of the end-on mode, the interaction with $\mathrm{Cr}$ makes the $\mathrm{C}-\mathrm{O}$ bond polarizability increasing, as illustrated for $\mathrm{Cr}(\mathrm{CO})_{6}$ in Figure 1, where, for comparison, the uncoordinated $\mathrm{CO}$ molecule is also shown. $\alpha_{\mathbf{C}-\mathbf{O}}$ is in fact more than two times larger in the complex than in isolation. This is particularly relevant when analyzing the reactivity of $\mathrm{M}(\mathrm{CO})_{\mathrm{n}}$ species. In fact, these complexes are often employed in catalytical processes, like the hydro-formylation reaction [20], where $\mathrm{CO}$ insertion into a metalalkyl bond occurs in a metal carbonyl catalyst after coordination of an olefin. The CO-insertion step requires a substantial repolarization of the CO ligand and an enhancement of its electrophilicity.

It is interesting that the coordination to a cationic, instead of a zerovalent metal, changes quite substantially the bond polarizabilities. In $\left[\mathrm{Ag}(\mathrm{CO})_{2}\right]^{+}$, the metal is much less polarizable, despite having more electrons, both because of its +1 oxidation state and of the unavailable low energy unoccupied d-orbitals, at variance from $\mathrm{Cr}(0)$. Consequently, $\mathrm{Ag}-\mathrm{C}$ bond is less polarizable than $\mathrm{Cr}-\mathrm{C}$ and, very importantly, so it is for the C-O bond (13.7 $\mathrm{Bohr}^{3}$ in uncoordinated $\mathrm{CO} ; 19.2 \mathrm{Bohr}^{3}$ in the $\mathrm{Ag}^{+}$complex; $33.4 \mathrm{Bohr}^{3}$ in $\mathrm{Cr}^{0}$ complex). 
It is also interesting to see what happens to hydride species, where one $\mathrm{CO}$ ligand is replaced by a $\mathrm{H}^{-}$. $\left[\mathrm{HM}(\mathrm{CO})_{\mathrm{n}}\right]^{\mathrm{q}-}$ are in fact the active species in the hydroformilation process (in particular for $\mathrm{M}=\mathrm{Co}$ ). While $\mathrm{H}$ atoms are normally very weakly polarizable, the hydride is in fact quite polarizable especially in the direction of the metal, as we can see for $\left[\mathrm{Cr}(\mathrm{CO})_{5} \mathrm{H}\right]^{-}$in Figure 2. In fact, the $\mathrm{Cr}-\mathrm{H}$ bond has a polarizability of $42.8 \mathrm{Bohr}^{3}$. This is actually due to two factors: one is the negative charge of the $\mathrm{H}$, the other is again the interaction with $\mathrm{Cr}$. Compared to neutral $\mathrm{Cr}(\mathrm{CO})_{6}$, the $\mathrm{Cr}-\mathrm{C}$ bond polarizabilities are slightly larger, but for $\mathrm{Cr}-\mathrm{C}$ trans to $\mathrm{Cr}-\mathrm{H}$, which is significantly smaller. $\mathrm{C}-\mathrm{O}$ polarizabilities are not particularly affected, being only slightly smaller than for $\mathrm{Cr}(\mathrm{CO})_{6}$.

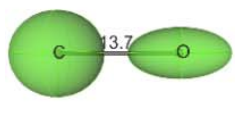

$\alpha_{\mathrm{xx}}{ }^{\mathrm{Mol}}=8.9 \mathrm{Bohr}^{3}$ $\alpha_{z z}{ }^{\mathrm{Mol}}=13.7 \mathrm{Bohr}^{3}$ $\alpha_{\text {iso }}{ }^{\mathrm{Mol}}=10.5 \mathrm{Bohr}^{3}$
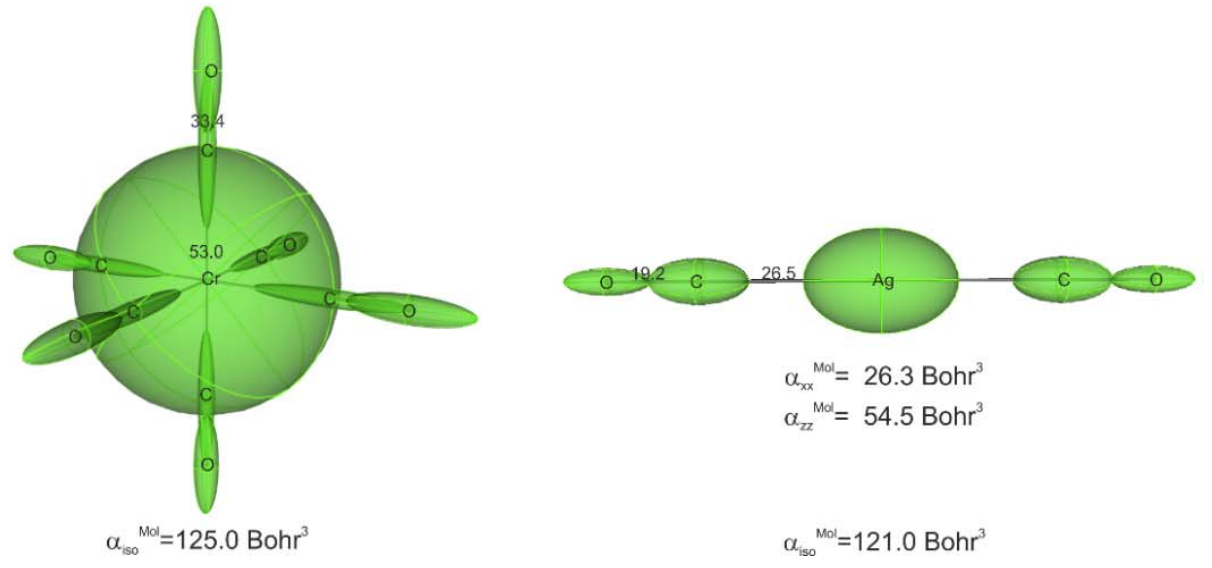

$\alpha_{x x}{ }^{\text {Mol }}=26.3$ Bohr $^{3}$ $\alpha_{\mathrm{zz}}{ }^{\mathrm{Mol}}=54.5 \mathrm{Bohr}^{3}$

$\alpha_{\text {iso }}{ }^{\mathrm{Mol}}=121.0 \mathrm{Bohr}^{3}$

Figure 1 The distributed atomic polarizabilities in isolated $\mathrm{CO}\left(\mathrm{C}_{\infty \mathrm{v}}\right.$, left $), \mathrm{Cr}(\mathrm{CO})_{6}\left(\mathrm{O}_{\mathrm{h}}\right.$, center $)$ and $\left[\mathrm{Ag}(\mathrm{CO})_{2}\right]^{+}$ ( $\mathrm{D}_{\infty \mathrm{h}}$, right), calculated at B3LYP/DGDZVP level of theory. $\mathrm{z}$ is parallel to the $\mathrm{C}_{\infty}$ axis for $\mathrm{CO}$ and $\left[\mathrm{Ag}(\mathrm{CO})_{2}\right]^{+}$. The values of bond and molecular polarizabilities are reported (in Bohr ${ }^{3}$ ). The scale factor used to represent the polarizabilities is $0.4 \mathrm{Bohr}^{-2}$. B3LYP/6-311+G(2d,2p) results are given in Table 1 .

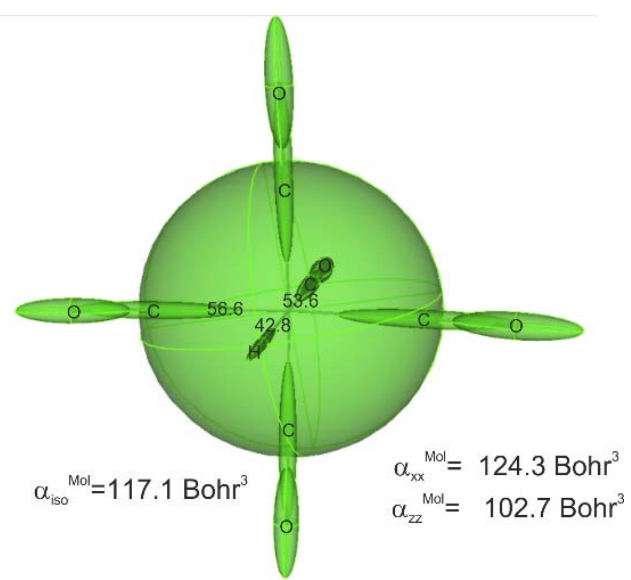

Figure 2 The distributed atomic polarizabilities in $\left[\mathrm{Cr}(\mathrm{CO})_{5} \mathrm{H}\right]^{-}$, in $\mathrm{C}_{4 \mathrm{v}}$ symmetry ( $\mathrm{z}$ axis parallel to $\mathrm{C}_{4}$ ), calculated at B3LYP/6-311+G(2d,2p) level. For sake of clarity, only $\mathrm{Cr}-\mathrm{C}$ and $\mathrm{Cr}-\mathrm{H}$ bond polarizabilities are reported. The scale factor used to represent the polarizabilities is 0.4 Bohr ${ }^{-2}$. At B3LYP/DGZVDP level, $\mathrm{Cr}-\mathrm{H}$ polarizability is $42.8 \mathrm{Bohr}^{3}$, $\mathrm{Cr}-\mathrm{C}$ (trans) is $53.6 \mathrm{Bohr}^{3}$ and $\mathrm{Cr}-\mathrm{C}($ cis $)$ is $56.6 \mathrm{Bohr}^{3}$. 


\subsection{Metal-olefins}

Another interesting class of compounds are the metal-olefins. At variance from $\mathrm{CO}$, olefins typically coordinate side-on, which implies simultaneous and symmetrical bonding of the two $\mathrm{C}$ atoms. Electron density studies have well illustrated that donation and back-donation are clearly visible through a QTAIM topological analysis [21]. Because of the side-on coordination, the C polarizabilities are re-oriented toward the metal and therefore away from the $\mathrm{C}=\mathrm{C}$ bond, unlike the uncoordinated olefin. As an example, $\mathrm{Cr}(\mathrm{CO})_{5}\left(\mathrm{C}_{2} \mathrm{H}_{4}\right)$ is illustrated in Figure 3. The Cr polarizability is now a tri-axial ellipsoid: the longest axis $\left(37.1 \mathrm{Bohr}^{3}\right)$ is in direction of the molecular 2-fold axis (hence towards the olefin) and the two smaller axes are in direction parallel to the $\mathrm{C}=\mathrm{C}$ bond $\left(32.2 \mathrm{Bohr}^{3}\right)$ and perpendicular to it $\left(33.8 \mathrm{Bohr}^{3}\right)$. The reason of this behaviour is the presence of two coordinating atoms on the olefin site, instead of one, as for hydride or carbonyl complexes. Although Carbon is less polarizable than Oxygen, a side-on coordination of two $\mathrm{C}$ atoms affects the $\mathrm{Cr}$ polarizability more than the end-on coordination of one $\mathrm{C}$ atom with the $\mathrm{O}$ atom quite far from the metal.

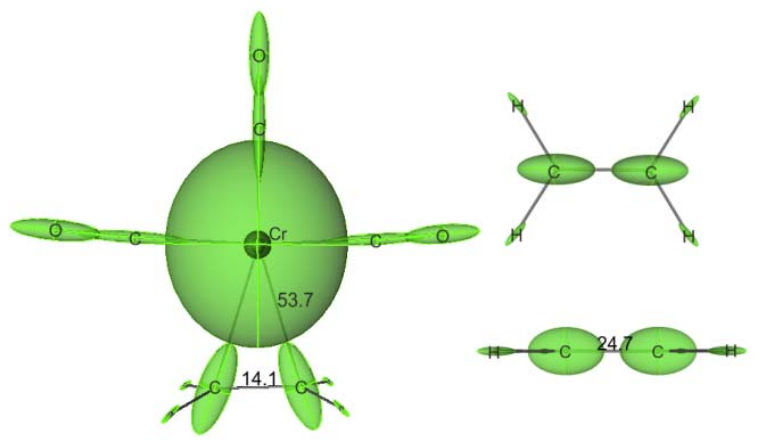

Figure 3 Distributed atomic polarizabilities in $\mathrm{Cr}(\mathrm{CO})_{5}\left(\mathrm{C}_{2} \mathrm{H}_{4}\right)\left(\mathrm{C}_{2 \mathrm{v}}\right.$, left) and in free $\mathrm{C}_{2} \mathrm{H}_{4}\left(\mathrm{D}_{2 \mathrm{~h}}\right.$, top and side view of the molecule, on the right), calculated at B3LYP/6-311+G(2d,2p) level of theory. The bond polarizabilities of $\mathrm{Cr}-\mathrm{C}_{\text {olefin }}$ and C-C are reported (in Bohr ${ }^{3}$ ). Scaling factor for drawing: $0.4 \mathrm{Bohr}^{-2}$.

While for the carbonyl ligand the main effect of coordination is a huge increase of the C-O bond polarizability, in olefins the most important and evident feature is the re-orientation of the $\mathrm{C}$ polarizability, which makes the attack of a nucleophile more feasible because it can occur almost perpendicularly to the $\mathrm{C}=\mathrm{C}$ bond. In uncoordinated olefins, instead, the best approach would be opposite to the $\mathrm{C}=\mathrm{C}$ bond, but the $\mathrm{H}$ atoms hinder it.

Noteworthy, the analysis of the atomic polarizabilities is complementary to the analysis of the electrostatic potential or the electron density Laplacian, which are two indicators of the potentially favourable sites for attack by hard nucleophiles. In Figure 4, one see that in fact the coordination to the metal also modifies the electrostatic potential around the $\mathrm{C}_{2} \mathrm{H}_{4}$ molecule. Upon coordination, the 
regions of negative potential around the double bond disappear, which means that an incoming nucleophile is no longer experiencing an electrostatic repulsion. However, one should consider that the potential around the uncoordinated ethylene is only slightly negative ( $\phi(\mathbf{r})$ is always above -0.025 a.u.) and a path of positive potential leading to $\mathrm{C}$ atom is always available. Thus, the actual change of electrostatic potential due to coordination to $\mathrm{Cr}$ is not so large, at variance from the abrupt change occurring to the atomic polarizabilities. As an example, let's consider the Wacker acethaldeide synthesis [22] where $\mathrm{H}_{2} \mathrm{O}$ or $(\mathrm{OH})^{-}$attack ethylene coordinated to a $\mathrm{Pd}$ complex. The enhanced polarizability in the $\mathrm{C}-\mathrm{M}$ direction $\left(>15 \mathrm{Bohr}^{3}\right.$ at the $\mathrm{C}$ site) is the main reason of this attack, especially if compared with the corresponding polarizability perpendicular to the $\mathrm{C}=\mathrm{C}$ plane in the uncoordinated ethylene $\left(8 \mathrm{Borh}^{3}\right)$.
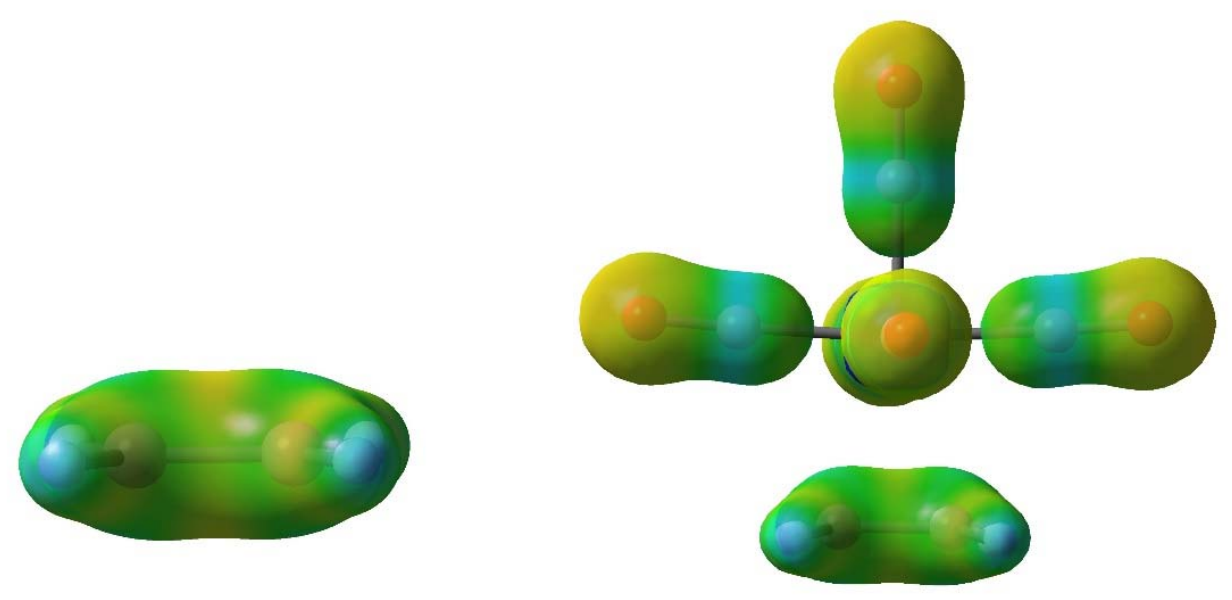

Figure 4 The electrostatic potential of $\mathrm{C}_{2} \mathrm{H}_{4}$ in isolation (left) and in $\mathrm{Cr}(\mathrm{CO})_{5}\left(\mathrm{C}_{2} \mathrm{H}_{4}\right)$ complex (right) calculated at B3LYP/6-311+G(2d,2p) level of theory. Cr-C bonds are omitted for clarity. For both pictures, the potential is plot with the same colour scale between -0.1 (red) and +1.0 a.u (blue) on the electron density isosurfaces of 0.1 a.u.

Another example of olefin coordination is a tetra-coordinated olefin complex, $\mathrm{Ni}(\mathrm{COD})_{2}(\mathrm{COD}=1,5$ cyclo-octadiene), presented in Figure 5. An experimental electron density study [21] analysed the QTAIM topology and demonstrated how this complexation leaves clear fingerprints in the molecular graph. At the same time, the Laplacian distribution around the ligand show that the electron density of the olefin is quite shifted in direction of the metal, a sign that the ligand have become more electrophilic. The coordination occurs through four $\mathrm{C}=\mathrm{C}$ disposed almost tetrahedrically around the $\mathrm{Ni}$ atom, although the molecular symmetry is much smaller $\left(\mathrm{D}_{2}\right)$, due to the geometry of the cyclic ligand. The ellipsoid representing the Ni polarizability tensor is quite aspherical, because stretched along the main $\mathrm{C}_{2}$ axis in direction of the two ligands where, apart from the olefinic $\mathrm{C}$ atoms, there are other polarizable $\mathrm{C}$ atoms, that play a role even if not directly bounded to the metal. Along the molecular 
$\mathrm{C}_{2}$ ' axes, no atom is present and the corresponding components of the Ni polarizability tensor are very small (ca. 9 Bohr $^{3}$ compared to ca. 50 Bohr $^{3}$ of the largest component).

The Ni-C polarizabilities are quite smaller than $\mathrm{Cr}-\mathrm{C}$ ones in $\mathrm{Cr}(\mathrm{CO})_{5} \mathrm{C}_{2} \mathrm{H}_{4}\left(42.9\right.$ vs $\left.53.7 \mathrm{Bohr}^{3}\right)$. This is in part due to the metal, although $\mathrm{Ni}$ and $\mathrm{Cr}$ in zero oxidation state have very similar atomic polarizabilities [23], Cr being only slightly larger. The difference is also due to the kind of ligand. A calculation on $\mathrm{Ni}\left(\mathrm{C}_{2} \mathrm{H}_{4}\right)_{4}$ in $\mathrm{D}_{2 \mathrm{~d}}$ symmetry, which is also characterized by 4 ligands disposed almost tetrahedrically around the metal, predicts a bond polarizability of $46 \mathrm{Bohr}^{3}$. The smaller Ni-C polarizability for cyclic olefins is in keeping with the known nucleophilic susceptibility series [24], which rank acyclic olefins with an even hapticity as the most reactive.

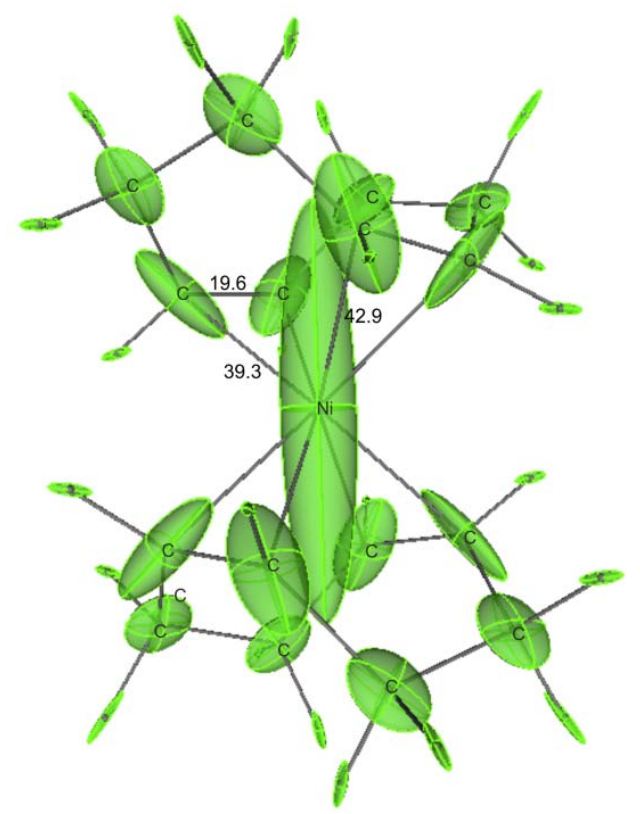

Figure 5 Distributed atomic polarizabilities in $\mathrm{Ni}(\mathrm{COD})_{2}$, calculated at B3LYP/6-311+G(2d,2p) level of theory. Bond polarizabilities for two non-equivalent $\mathrm{Ni}-\mathrm{C}$ bonds and the $\mathrm{C}=\mathrm{C}$ bond are reported (in Bohr ${ }^{3}$ ). Scaling factor $0.3 \mathrm{Bohr}^{-2}$. Note that bonds are drawn between atoms connected by a bond path; however, the lines are simply straight vectors and not the exact representation of the bond paths.

\subsection{Agostic interactions}

The agostic bond, where a $\mathrm{C}-\mathrm{H}$ or a $\mathrm{Si}-\mathrm{H}$ bond approaches end on a transition metal producing a drastic changes of properties for $\mathrm{H}$ atom, is among the most interesting interactions in organometallic chemistry. This interaction is in fact extremely important because it enables activation of a bond like $\mathrm{C}-\mathrm{H}$, which is non-polar, weakly polarizable and very strong. Many electron density studies based on QTAIM [25], as well as theoretical or spectroscopic investigations, have analysed these interactions, whose nature remained quite ambiguous for long time. The agostic bond is associated with a large delocalization that involves the entire alkyl group typically attached to the metal via a strong M-C bond.

Here, we report only a single example, namely $\left[\mathrm{Ni}(\mathrm{dhpe})\left(\mathrm{C}_{2} \mathrm{H}_{5}\right)\right]^{+}$(dhpe $\left.=\mathrm{PH}_{2} \mathrm{CH}_{2} \mathrm{CH}_{2} \mathrm{PH}_{2}\right)$, a model compound similar to that investigated experimentally by Scherer et al. [25e]. A bond path is found 
between $\mathrm{Ni}$ and $\mathrm{H}$ (see Figure 6), although this is not a necessary conditions to identify an agostic interaction [25a] and was actually missing in the experimental study, after a careful re-examination of the model. What is particularly evident, however, is the quite large polarizability of the $\mathrm{H}$, oriented toward the metal instead of the $\mathrm{C}$ atom. In general $\mathrm{H}$ atoms are weakly polarizable with an extremely large anisotropy of their tensors (see all the other C-H bonds in Figure 6, for example). Noteworthy, the large reorientation of the agostic hydrogen polarizability would occur even in the absence of a bond path to $\mathrm{Ni}$, given that the bond dipole contribution of $\mathrm{Ni}-\mathrm{H}$ is in fact very small, because this interaction is associated with an extremely small electron density at the BCP (hence a large $\lambda(\mathrm{Ni} \mid \mathrm{H})$ ). In fact, when $\lambda$ is large, the contribution of the bond to the atomic dipole is small.

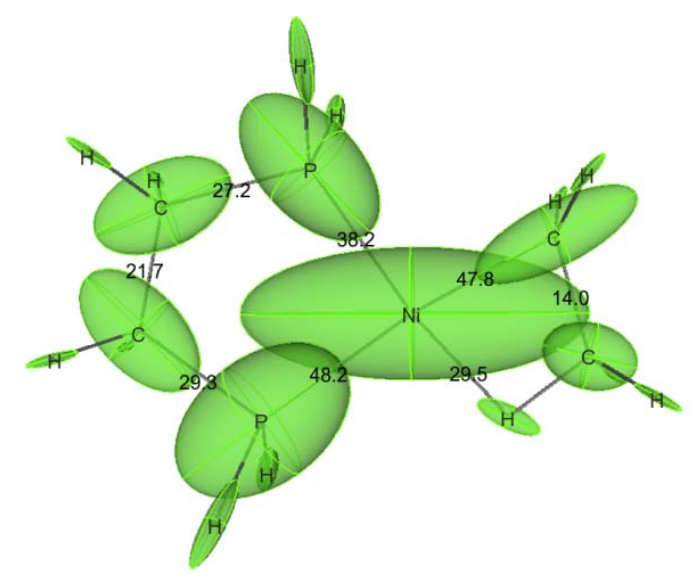

Figure 6 Distributed atomic polarizabilities in $\left[\mathrm{Ni}(\mathrm{dhpe})\left(\mathrm{C}_{2} \mathrm{H}_{5}\right)\right]^{+}$. Bond polarizabilities are given in Bohr ${ }^{3}$. Scaling factor $0.4 \mathrm{Bohr}^{-2}$. Note that bonds are drawn between atoms connected by a bond path; however, the lines are simply straight vectors and not the exact representation of the bond paths.

\subsection{Metal-Metal bonds}

One of the most interesting classes of organometallic molecules are the metal clusters, where metal atoms are bonded to each other directly or through bridging ligands. In such clusters, the direct connection between two highly polarizable atoms produce a very large polarizability along the M-M direction. This was anticipated by Parr [1] who proposed it as one reason of the overall stability of the dimer. The enormous enhancement is due to pure electrostatic reasons: two polarizable charge distribution affect each other under an applied electric field, because the field-induced dipole moments of one distribution increase the electric field experienced by the other distribution (and vice versa). Therefore, the two zerovalent transition metals mutually increase their polarizability along the direction of their connection. In fact, a large $\alpha_{\mathrm{Mn}-\mathrm{Mn}}$ is calculated for $\mathrm{Mn}_{2}(\mathrm{CO})_{10}$ where a direct bond path connects the two $\mathrm{Mn}$ atoms (Figure 7a). The same occurs for the $\mathrm{C}_{\mathrm{s}}$ isomer of $\mathrm{Co}_{2}(\mathrm{CO})_{8}$ (Figure $7 \mathrm{~b})$ although a direct bond path between the metals is not calculated. The two $\mathrm{Co}(\mathrm{CO})_{3}$ subunit are not co-axial given the disposition of the two bridging ligands. Thus, the two Co polarizabilities are also not co-axial but they point towards the "missing" bridging carbonyl. Interestingly, this is the region of a possible curved Co-Co bond path, whose presence was debated for long time. 
The polarizability of the bridging carbonyl is very peculiar.in contrast to atomic polarizabilities of terminal CO groups, which are very much prolated in the direction of the bonded Co atom, the bridging $\mathrm{C}$ atoms have polarizability ellipsoid similar to disks "stretched" by the two Co and the oxygen atoms. Perpendicularly to the plane defined by Co-C-Co, the tensor component is extremely small.

It is noteworthy that the equatorial carbonyls of $\mathrm{Mn}_{2}(\mathrm{CO})_{10}$, albeit not directly bonded to the vicinal $\mathrm{Mn}$, are quite distorted in that direction so that the principal component of their tensors is not parallel to $\mathrm{Mn}-\mathrm{C}$, as one would expect for a simple metal complex (as $\left.\mathrm{Cr}(\mathrm{CO})_{6}\right)$. The metamorphosis of the carbonyl ligand from terminal to bridging was already described in terms of molecular graph, Laplacian and electron delocalization indices in [26], showing that a rather continuous bonding is ongoing. The shape of the polarizabilities of equatorial Carbons reinforces the idea that they play an important role in the bonding even in the absence of a direct bond path.

Another interesting stereochemistry of M-M bonded system is the recently discussed off-axis bond, found in $\mathrm{Mn}_{2}(\mathrm{CO})_{10}$ at high pressure [27]. The two $\mathrm{Mn}(\mathrm{CO})_{5}$ units are forced to be no longer co-axial so that the apical carbonyls and the Mn-Mn bonds are not parallel (see Figure 7c). Interestingly, although the Mn-Mn bond decreases, as expected from a compression mechanism, the bond polarizability is actually smaller ( 117 vs $138 \AA$ ). The major axis of the polarizability tensors of the Mn is parallel to the Mn-Mn bond and therefore inclined with respect to the apical Mn-C bonds, meaning that Mn-Mn bond is overwhelming. 


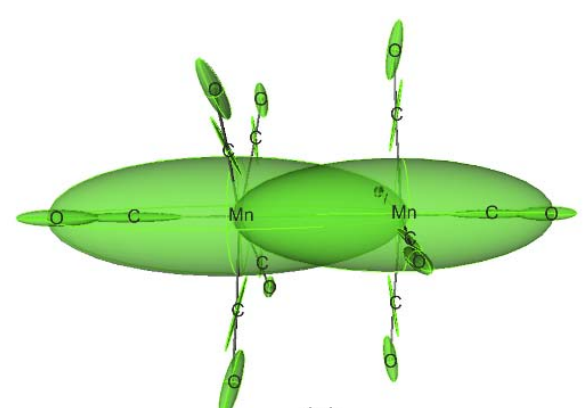

(a)
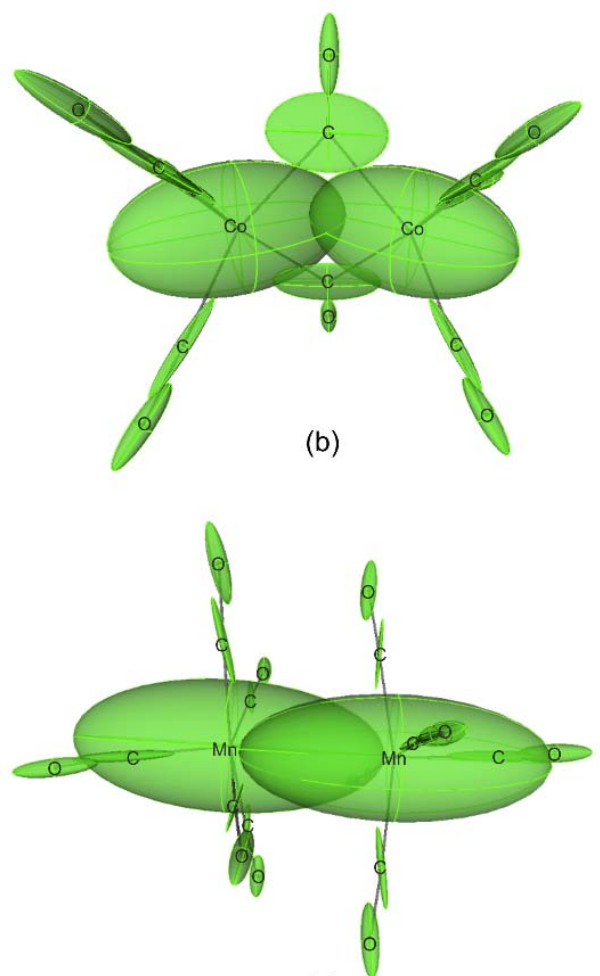

(c)

Figure 7 The distributed atomic polarizabilities in $\mathrm{Mn}_{2}(\mathrm{CO})_{10}$ (a) $\left(\mathrm{D}_{4 \mathrm{~d}}\right.$, gas phase stable isomer), $\mathrm{Co}_{2}(\mathrm{CO})_{8}(\mathrm{~b})$ $\left(\mathrm{C}_{2 \mathrm{v}}\right.$, one of the three gas phase isomers and the only solid state stable isomer) and in the "off-axis" $\mathrm{Mn}_{2}(\mathrm{CO})_{10}$ (c) $\left(\mathrm{C}_{2}\right.$ symmetry, stable in the solid state, ) Scaling factor $0.3 \mathrm{Bohr}^{-2}$. Note that bonds are drawn between atoms connected by a bond path; however, the lines are simply straight vectors and not the exact representation of the bond paths.

\section{Conclusions}

In this paper, we have presented the application of the distributed atomic polarizabilities to the analysis of the nature and the reactivity of some well-known chemical bonds in organometallic chemistry. The analysis of polarizabilities is in a way a visualization of the available mixing of ground and excited states for a molecule. In fact, instead of finite field numerical differentiations as in our approach, one could calculate polarizabilities via a sum over states, which would make the connection with chemical softness more obvious. The possibility to partition the polarizability into atomic 
contributions visualizes the contribution of each atom to excited states and therefore enable to somewhat localize sites of larger softness.

Molecular recognition processes, that anticipate chemical reactions, are normally predicted based only on the electrostatic potential that addresses sites where positive or negative charges are in excess. This approach, however, is valid mainly for hard nucleophiles or electrophiles, but may be less informative when dealing with soft species. Therefore, we propose that distributed atomic polarizabilities may be complementarily analysed to anticipate the reactivity of a given species, or, as in this paper, to investigate the role of metal coordination in enhancing the electrophilic or nucleophilic susceptibility. The terms "charge-controlled" or "orbital-controlled" reactions are less common nowadays but could be in fact associated with this dual visualization: electrostatic potential and electric polarizabilities, respectively.

In the future, we plan to derive distributed atomic hyperpolarizabilities, although the finite field method may give more convergence problems. This would enable a more accurate and potentially quantitative analysis, even in the presence of stronger fields.

Moreover, although we have used so far only the QTAIM partitioning to compute atomic polarizabilities, other schemes might be suitable and will be tested in the future.

Finally, DFT methods have been used here to compute the electron density distributions; however, more accurate electron correlation methods (e.g., configuration interaction or perturbation methods) could be adopted, although at higher computational costs.

\section{ACKNOWLEDGMENT}

We thank the Swiss National Science Foundation for financial support (project Nr. 141271). We thank Dr. D. Pérez for preparation of the ViewTensor graphical routine within the program PolaBer. 
Table 1. Summary of the most relevant bond polarizabilities calculated for the complexes reported in the text. All entries are in atomic units. Pol A- and Pol -B are the bond polarizability of each atom; Pol A-B is the total bond polarizability. When important, the symmetry of the compound is also reported.

\begin{tabular}{|c|c|c|c|c|c|}
\hline Compound & Bond A-B & Basis Set & Pol A- & Pol -B & Pol A-B \\
\hline CO & $\mathrm{C}-\mathrm{O}$ & $\begin{array}{l}\text { DGDZVP } \\
6-311+G(2 d, 2 p)\end{array}$ & $\begin{array}{l}6.39 \\
7.27\end{array}$ & $\begin{array}{l}7.33 \\
8.04\end{array}$ & $\begin{array}{l}13.72 \\
15.31\end{array}$ \\
\hline $\mathrm{Cr}(\mathrm{CO})_{6}$ & $\begin{array}{l}\mathrm{C}-\mathrm{O} \\
\mathrm{Cr}-\mathrm{C}\end{array}$ & $\begin{array}{l}\text { DGDZVP } \\
6-311+G(2 d, 2 p) \\
\text { DGDZVP } \\
6-311+G(2 d, 2 p)\end{array}$ & $\begin{array}{l}19.65 \\
19.28 \\
19.65 \\
19.28\end{array}$ & $\begin{array}{l}13.76 \\
14.62 \\
33.41 \\
33.61\end{array}$ & $\begin{array}{l}33.41 \\
33.90 \\
53.06 \\
52.89\end{array}$ \\
\hline $\mathrm{Cr}(\mathrm{CO})_{5} \mathrm{H}$ & $\begin{array}{l}\mathrm{C}-\mathrm{O} \text { (trans) } \\
\mathrm{C}-\mathrm{O} \text { (cis) } \\
\mathrm{Cr}-\mathrm{C} \text { (trans) } \\
\mathrm{Cr}-\mathrm{C} \text { (cis) } \\
\mathrm{Cr}-\mathrm{H}\end{array}$ & $\begin{array}{l}\text { DGDZVP } \\
\text { 6-311+G(2d,2p) } \\
\text { DGDZVP } \\
\text { 6-311+G(2d,2p) } \\
\text { DGDZVP } \\
\text { 6-311+G(2d,2p) } \\
\text { DGDZVP } \\
\text { 6-311+G(2d,2p) } \\
\text { DGDZVP } \\
\text { 6-311+G(2d,2p) }\end{array}$ & $\begin{array}{l}21.33 \\
19.88 \\
21.17 \\
19.97 \\
32.29 \\
29.00 \\
35.48 \\
34.24 \\
32.29 \\
29.00\end{array}$ & $\begin{array}{l}14.91 \\
13.64 \\
14.87 \\
13.65 \\
21.33 \\
19.88 \\
21.17 \\
19.97 \\
10.48 \\
11.72\end{array}$ & $\begin{array}{l}36.24 \\
33.52 \\
36.04 \\
33.62 \\
53.62 \\
48.88 \\
56.65 \\
54.21 \\
42.77 \\
40.72\end{array}$ \\
\hline$\left[\mathrm{Ag}(\mathrm{CO})_{2}\right]^{+}$ & $\begin{array}{l}\mathrm{Ag}-\mathrm{C} \\
\mathrm{C}-\mathrm{O}\end{array}$ & $\begin{array}{l}\text { DGDZVP } \\
\text { DGDZVP }\end{array}$ & $\begin{array}{l}16.15 \\
10.36\end{array}$ & $\begin{array}{l}10.36 \\
8.81\end{array}$ & $\begin{array}{l}25.51 \\
19.17\end{array}$ \\
\hline $\mathrm{C}_{2} \mathrm{H}_{4}$ & $\begin{array}{l}\mathrm{C}-\mathrm{C} \\
\mathrm{C}-\mathrm{H}\end{array}$ & $\begin{array}{l}6-311+G(2 d, 2 p) \\
6-311+G(2 d, 2 p)\end{array}$ & $\begin{array}{c}12.35 \\
6.96\end{array}$ & $\begin{array}{c}12.35 \\
4.63\end{array}$ & $\begin{array}{l}24.70 \\
11.59\end{array}$ \\
\hline $\mathrm{Cr}(\mathrm{CO})_{5}\left(\mathrm{C}_{2} \mathrm{H}_{4}\right)$ & $\begin{array}{l}\mathrm{C}-\mathrm{O} \text { (trans) } \\
\mathrm{C}-\mathrm{O} \text { (cis) })^{(\mathrm{a})} \\
\mathrm{Cr}-\mathrm{CO} \text { (trans) } \\
\mathrm{Cr}-\mathrm{CO}(\text { cis) } \\
\mathrm{Cr}^{(\mathrm{a})} \mathrm{C}_{2} \mathrm{H}_{4} \\
\mathrm{C}-\mathrm{C}\end{array}$ & $\begin{array}{l}6-311+G(2 d, 2 p) \\
6-311+G(2 d, 2 p) \\
6-311+G(2 d, 2 p) \\
6-311+G(2 d, 2 p) \\
6-311+G(2 d, 2 p) \\
6-311+G(2 d, 2 p)\end{array}$ & $\begin{array}{c}19.18 \\
18.96 \\
37.13 \\
33.00 \\
36.73 \\
7.06\end{array}$ & $\begin{array}{c}15.06 \\
14.81 \\
19.17 \\
18.96 \\
17.03 \\
7.06\end{array}$ & $\begin{array}{l}34.24 \\
33.77 \\
56.30 \\
51.96 \\
53.76 \\
14.12\end{array}$ \\
\hline $\mathbf{N i}(\mathrm{COD})_{2}\left(\mathrm{D}_{2}\right)$ & $\begin{array}{l}\mathrm{Ni}-\mathrm{C} \\
\mathrm{Ni}-\mathrm{C} \\
\mathrm{C}-\mathrm{C}^{(b)}\end{array}$ & $\begin{array}{l}6-311+G(2 d, 2 p) \\
6-311+G(2 d, 2 p) \\
6-311+G(2 d, 2 p)\end{array}$ & $\begin{array}{l}29.4 \\
26.1 \\
10.2\end{array}$ & $\begin{array}{c}13.5 \\
13.2 \\
9.4\end{array}$ & $\begin{array}{l}42.9 \\
39.3 \\
19.6\end{array}$ \\
\hline$\left[\mathrm{Ni}(\mathrm{dhpe})\left(\mathrm{C}_{2} \mathrm{H}_{5}\right)\right]^{+}$ & $\begin{array}{l}\mathrm{Ni}-\mathrm{C} \\
\mathrm{Ni}-\mathrm{H} \\
\mathrm{Ni}-\mathrm{P}(\text { trans })^{(\mathrm{c})} \\
\mathrm{Ni}-\mathrm{P}\left(\text { cis }^{(\mathrm{c})}\right.\end{array}$ & $\begin{array}{l}6-311+\mathrm{G}(2 \mathrm{~d}, 2 \mathrm{p}) \\
6-311+\mathrm{G}(2 \mathrm{~d}, 2 \mathrm{p}) \\
6-311+\mathrm{G}(2 \mathrm{~d}, 2 \mathrm{p}) \\
6-311+\mathrm{G}(2 \mathrm{~d}, 2 \mathrm{p})\end{array}$ & $\begin{array}{l}30.18 \\
23.33 \\
20.34 \\
27.89\end{array}$ & $\begin{array}{c}17.60 \\
6.16 \\
17.89 \\
20.28\end{array}$ & $\begin{array}{l}47.78 \\
29.49 \\
38.23 \\
48.17\end{array}$ \\
\hline $\operatorname{Mn}_{2}(\mathbf{C O})_{10}\left(\mathrm{D}_{4 \mathrm{~d}}\right)$ & $\begin{array}{l}\text { Mn-Mn } \\
\text { Mn-C equatorial } \\
\text { Mn-C axial } \\
\text { C-O equatorial } \\
\text { C-O axial }\end{array}$ & $\begin{array}{l}6-311+\mathrm{G}(2 \mathrm{~d}, 2 \mathrm{p}) \\
6-311+\mathrm{G}(2 \mathrm{~d}, 2 \mathrm{p}) \\
6-311+\mathrm{G}(2 \mathrm{~d}, 2 \mathrm{p}) \\
6-311+\mathrm{G}(2 \mathrm{~d}, 2 \mathrm{p}) \\
6-311+\mathrm{G}(2 \mathrm{~d}, 2 \mathrm{p})\end{array}$ & $\begin{array}{l}69.22 \\
24.39 \\
69.22 \\
14.23 \\
19.22\end{array}$ & $\begin{array}{l}69.22 \\
14.06 \\
19.22 \\
11.24 \\
14.41\end{array}$ & $\begin{array}{l}138.44 \\
38.45 \\
88.44 \\
25.47 \\
33.63\end{array}$ \\
\hline $\mathrm{Mn}_{2}(\mathrm{CO})_{10}\left(\mathrm{C}_{2}\right)$ & $\begin{array}{l}\text { Mn-Mn } \\
\text { Mn-C equatorial }^{(d)} \\
\text { Mn-C axial } \\
\text { C-O equatorial } \\
\text { C-O axial }\end{array}$ & $\begin{array}{l}6-311+G(2 d, 2 p) \\
6-311+G(2 d, 2 p) \\
6-311+G(2 d, 2 p) \\
6-311+G(2 d, 2 p) \\
6-311+G(2 d, 2 p)\end{array}$ & $\begin{array}{l}58.47 \\
25.06 \\
57.64 \\
13.81 \\
19.08\end{array}$ & $\begin{array}{l}58.47 \\
14.00 \\
19.24 \\
12.22 \\
15.98 \\
\end{array}$ & $\begin{array}{l}116.95 \\
39.06 \\
76.86 \\
26.03 \\
35.06 \\
\end{array}$ \\
\hline
\end{tabular}




\section{REFERENCES}

${ }^{1}$ R. G. Pearson, Hard and Soft Acids and Bases, J. Am. Chem. Soc. 82 (1963) 3533-3539.

${ }^{2}$ R. G. Parr, R., W. Yang, Density Functional theory of Atoms and Molecules, Oxford University Press, Oxford, 1989.

${ }^{3}$ R. G. Parr, R. G. Pearsons, Absolute Hardness: Companion Parameter to Absolute Electronegativity, J. Am. Chem. Soc., 105 (1983) 7512-7516

${ }^{4}$ R. G. Pearson, Chemical Hardness and Density functional Theory, J. Chem. Sci. 117 (2005) 369-377.

5 (a) L. Komorowski, J. Lipiński, P. Szarek, Polarization justified Fukui functions, J. Chem. Phys. 131 (2009), 124120; (b) L. Komorowski, J. Lipiński, P. Szarek, P. Ordon, Polarization justified Fukui functions: The theory and applications for molecules, J. Chem. Phys. 135 (2011), 014109.

${ }^{6}$ R.F.W. Bader, Atoms in Molecules: A Quantum Theory, Oxford University Press, Oxford, 1990.

${ }^{7}$ T.A. Keith, Atomic response properties, in C.F. Matta, R.J. Boyd (Eds.), The Quantum Theory of Atoms in Molecules: From Solid State to DNA and Drug Design, Viley-WCH, Weinheim, 2007, pp 61-95.

${ }^{8}$ (a) A. Krawczuk, D. Pérez, P. Macchi, J. Appl. Cryst. 47 (2014), doi:10.1107/S1600576714010838. (b) A. Krawczuk-Pantula, D. Pérez, K. Stadnicka, P. Macchi, Trans. Am. Crystallogr. Assoc. (2011) 1-25.

9 (a) A. J. Stone Distributed polarizabilities, Mol. Phys. 56 (1985) 1065-1082; (b) C. R. Le Sueuer, A. J. Stone Practical Schemes for distributed polareizabilities, Mol. Phys. 78 (1993) 1267-1291; (c) C. Hättig, G. Jansen, B. A. Hess, J. G. Ángyán, Topologically partitioned dynamic polarizabilities using the theory of atoms in molecules, Can. J. Chem. 74 (1996) 976-987; (d) K. M. Gough, M. M. Yacowar, R. H. Cleve, J. R. Dwyer, Analysis of molecular polarizabilities and polarizability derivatives in $\mathrm{H}_{2}, \mathrm{~N}_{2}, \mathrm{~F}_{2}, \mathrm{CO}$ and $\mathrm{HF}$ with the theory of atoms in molecules, Can. J. Chem., 74 (1996) 1139-1144; (e) A. Fkyerat, A. Guelzim, F. Baert, J. Zyss, A. Perigaud, Assessment of the polarizabilities of a nonlinear optical compound [N-(4-nitrophenyl)-(L)prolinol]from an experimental electronic density study, Phys. Rev. 53 (1996) 16236 - 16246; (f) F. Hamzaoui, A. Zanoun, G. Vergoten, The molecular linear polarizability from X-ray diffraction study. The case of 3-methyl 4-nitropyridine N-oxide (POM), J. Mol. Struct. 697 (2004) 17-22; (g) A. Chouaih, F. Hamzaoui, G. Vergoten, Capability of X-ray diffraction to the determination of the macroscopic linear susceptibility in a crystalline environment: the case of 3-Methyl 4-Nitropyridine N-oxide (POM), J. Mol. Struct. 738 (2005) 33-38; (h) I Soterasm C, Curutchet, A. Bidon-Chanal, F. Dehez, J. G. Ángyán, M. Orozco, C. Chipot, F. J. Luqze, Derivation of Distributed Models of Atomic Polarizability for Molecular Simulations, J. Chem. Theor. Comput., 3 (2007) 1901-1913.

${ }^{10}$ J. F. Nye, Physical Properties of Crystals, Oxford Science Publications, Oxford, 1957.

${ }^{11}$ G. Placzek, Rayleigh-Streuung und Raman-Effekt, in E. Marx (Ed.), Handbuch der Radiologie, Akademische Verlagsgesellschaft, Leipzig, Germany, 1934, pp. 205-374.

12 (a) D. A. Long, Intensities in Raman Spectra. I. A Bond Polarizability Theory, Proceedings of the Royal Society of London. Series A, Mathematical and Physical Sciences 217 (1953), 203-221; (b) R. Tubino, L. Piseri, Raman intensities in covalent crystals: A bond-polarizability approach, Phys. Rev. B (1960) 11, 5145-5152; (c) R.P. Smith, E.M. Mortensen, Bond and Molecular Polarizability Tensors. I. Mathematical Treatment of Bond Tensor Additivity, J. Chem. Phys. (1960) 32, 502-507; (d) S. Guha, J. Menéndez, J.B. Page, G.B. Adams, Empirical bond polarizability model for fullerenes, Phys. Rev. B 53 (1996), 13106-13114 
${ }^{13}$ M.J. Frisch et al., Gaussian 09, Revision D.01, Gaussian, Inc., Wallingford, CT, 2009.

${ }^{14}$ (a) A.D. Becke, Density-functional thermochemistry. III. The role of exact exchange, J. Chem. Phys. 98 (1993) 5648-5652. (b) C. Lee, W. Yang, R.G. Parr, Development of the Colle-Salvetti correlation-energy formula into a functional of the electron density, Phys. Rev. B 37 (1988) 785-789.

${ }^{15}$ A. D. McLean, G. S. Chandler, Contracted Gaussian-basis sets for molecular calculations. 1. 2nd row atoms, $\mathrm{Z}=11-18$, J. Chem. Phys., 72 (1980) 5639-48.

${ }^{16}$ N. Godbout, D. R. Salahub, J. Andzelm, E. Wimmer, Optimization of Gaussian-type basis sets for local spin density functional calculations. Part I. Boron through neon, optimization technique and validation, Can. J. Chem., 70 (1992) 560-71.

${ }^{17}$ Keith, T.A. (2013) AIMAll Version 13.05.06, TK Gristmill Software, Overland Park KS, USA.

18 (a) B. Rees, A. Mitschler, Electronic structure of chromium hexacarbonyl at liquid nitrogen temperature. 2. Experimental study (x-ray and neutron diffraction) of $\sigma$ and $\pi$ bonding, J. Am. Chem. Soc., 98 (1976) 79187924; (b) R. Bianchi, G. Gervasio, D. Marabello, Experimental charge density study of the Mn-Mn bond in Mn2(CO)10 at 120 K, Chemical Communications, (1998) 1535-1536; (c) P. Macchi, D. M. Proserpio, A. Sironi, Experimental electron density in a transition metal dimer: Metal-metal and metal-ligand bonds, J. Am. Chem. Soc., 120 (1998), 3429-13435; (d) L. J. Farrugia, P. R. Mallinson, B. Stewart, Experimental charge density in the transition metal complex Mn 2(CO)10: A comparative study, Acta Cryst., B59 (2003), 234-247; (e) P. Macchi, A. Sironi, Coord. Chem. Rev., Chemical bonding in transition metal carbonyl clusters: complementary analysis of theoretical and experimental electron densities., 238-239 (2003) 383-412; (f) L. J. Farrugia, C. Evans, Experimental X-ray charge density studies on the binary carbonyls $\mathrm{Cr}(\mathrm{CO})_{6}, \mathrm{Fe}(\mathrm{CO})_{5}$, and $\mathrm{Ni}(\mathrm{CO})_{4}, \mathrm{~J}$. Chem. Phys. A, 109 (2005) 8834-8848; (g) F. Cortés-Guzmán, R. F. W. Bader, Complementarity of QTAIM and MO theory in the study of bonding in donor-acceptor complexes, Coord. Chem. Rev., 249 (2005) 633-662.

${ }^{19}$ (a) M. J. S. Dewar, A review of $\pi$ Complex Theory, Bull. Soc. Chim. Fr., 18 (1951) C79; (b) J. Chatt, L. A. Duncanson, Olefin co-ordination compounds. Part III. Infra-red spectra and structure: attempted preparation of acetylene complexes, J. Chem. Soc., (1953) 2939.

${ }^{20}$ R. F. Heck, D. S. Breslow, The Reaction of Cobalt Hydrotetracarbonyl with Olefins, J. Am. Chem. Soc., 83 (1961) 4023-4027.

${ }^{21}$ P. Macchi, D. M. Proserpio, A. Sironi, Experimental electron density studies for investigating the metal $\pi$ ligand bond: the case of bis(1,5 cyclooctadiene) nickel. J. Am. Chem. Soc., 120 (1998) 1447-1455.

${ }^{22}$ G. W. Parshall, Homogeneous Catalysis, Wiley, New York, 1980.

${ }^{23}$ T. M. Miller, B. Bederson, Atomic and molecular polarizabilities - A review of recent advances, Advances in Atomic and Molecular Physics, 13 (1978) 1-55.

${ }^{24}$ J. E. Huheey, E. A. Keiter, R. L. Keiter, Inorganic Chemistry: principles of structure and reactivity, $4^{\text {th }}$ edition, Harper Collins, New York, 1993.

${ }^{25}$ (a) W. Scherer, G. S. McGrady, Agostic Interactions in d0 Metal Alkyl Complexes, Angew. Chem. Int Ed. Eng., 43 (2004) 1782 - 1806; (b) J. E. Barquera-Lozada, A. Obenhuber, C. Hauf, W. Scherer, On the Chemical Shifts of Agostic Protons 117 (2013) 4304-4315; (c) W. Scherer, V. Herz, C. Hauf, On the Nature of betaAgostic Interactions: A Comparison Between the Molecular Orbital and Charge Density Picture, Structure and Bonding, 146 (2012) 159-207; (d) W. Scherer, P. Sirsch, D. Shorokhov, M. Tafipolsky, G. S. McGrady, E. Gullo, Valence charge concentrations, electron delocalization an beta-agostic bonding in $\mathrm{d}(0)$ metal alkyl 
complexes, Chemistry, Eur. J., 9 (2003) 6057-6070; (e) W. Scherer, W. Hieringer, M. Spiegler, P. Sirsch, G. S. McGrady, A. J. Downs, A. Haaland, B. Pedersen, Characterisation of agostic interactions by a topological analysis of experimental and theoretical charge densities in $[\operatorname{EtTiCl} 3(\mathrm{dmpe})][\mathrm{dmpe}=1,2-$ bis(dimethylphosphino)ethane], Chem. Comm. (1998) 2471-2472

${ }^{26}$ P. Macchi, L. Garlaschelli, A. Sironi, Electron Density of Semi-Bridging Carbonyls. Metamorphosis of CO Ligands Observed via Experimental and Theoretical Investigations on $\left[\mathrm{FeCo}(\mathrm{CO})_{8}\right]^{-}$, J. Am. Chem. Soc., 124 (2002) 14173-14184.

27 P. Macchi, N. Casati, S. R. Evans, F. Gozzo, P. Simoncic, D. Tiana, "Off-axis" metal-metal bond in Mn2(CO)10 at high pressure, Chem. Commun. 2014, in the press DOI: 10.1039/C4CC04152F. 